\title{
A Fluorescence Molecular Tomography In-vivo Imaging System for Macro/Meso-Scale Subjects
}

Yiyong Tan* and Huabei Jiang*

J. Crayton Pruitt Family Department of Biomedical Engineering, University of Florida, Gainesville, FL 32611-6131, USA

\begin{abstract}
A novel quantitative fluorescence molecular tomography (FMT) imager for both macro and meso-scale animal experiments is developed. The image reconstruction algorithms are implemented in the framework of finite element method, while the experimental system is constructed using a non-contact, multi-angle full 360 degree transmission scheme. Shape-from-silhouette based volume carving approach is used to render the 3D models of the actual samples. With a free-space light propagation model, the readout from the CCD is converted into the photon density normal to sample surface in order to match the model-based tomographic reconstruction. For meso-scale subjects, a radiative transfer equation (RTE) based FMT reconstruction algorithm is adopted. For macro-scale subjects, a diffusion approximation (DA) based FMT reconstruction algorithm is adopted. Imaging results for both macro-scale and mesoscale subjects are presented to valid system performance.
\end{abstract}

Keywords: Fluorescence Molecular Tomography System; macro/ meso-scale

\section{Introduction}

FMT enables researchers to rapidly and easily obtain tomographic images of in vivo biomarkers with various advantages such as unprecedented depth penetration, no invasive via NIR light, wholebody animal imaging and true quantification in biological research, preclinical study and clinical diagnosis. In addition, fluorescence can be used in meso-scale to analyze the regulation and expression of genes, to locate proteins in cells and tissues, to follow metabolic pathways, to reveal neuron activity and to study the location, migration, communication of cells. Herein, we developed a 3D FMT system capable of imaging subject size in different magnitude as a low cost and handy solution of increasingly challenging biomedical research [1-5].

\section{Materials and Methods}

Figure1 is the CCD based non-contact continuous wave (CW) FMT system developed. The imaging system and diffuse optical tomography (DOT) guided FMT algorithm for arbitrarily shaped subjects is established and tested in previous study [6,7]. The system is capable of imaging both meso and macro scale animal with convenience. Software and reconstruction algorithm is developed to function for both meso and macro scale. For hardware, only CCD lens and sample stage need to be switch for different sample sizes (1-60135AD Z6000 W/12MM for Meso scale; Zoom 7000 for macro scale, Navitar, Inc., NY). The animals like rat, mice and Drosophila can be measured in their nature state; the data acquisition procedure and FMT reconstruction are unified for both big and tiny subjects.

In the imaging system, the excitation light can be delivered to the macro/meso size subject at multiple points in both $\mathrm{X}$ and $\mathrm{Y}$ directions via linear stages $(300 \mathrm{~nm}$ precision). For each source position, one set of excitation light data from the opposite side of the subject is recorded by a $1024 \times 1024$ pixels CCD camera (Princeton Instrument, Trenton, NJ). Emission light is collected by placing a band pass filter in front of the CCD. The system has been constructed such that multiangle transmission light can be collected for more accurate image reconstruction (four angles are used in most case with 0.5 arc second angle resolution). This is realized by rotating the subject three $90^{\circ}$ to collect transmission light at four different subject projections. The data acquisition and processing programs of instrument system are streamlined for maximum repeatability.

The well-known shape-from-silhouette 3D recovery approach was used to render the $3 \mathrm{D}$ model of the actual sample used [8]. In this

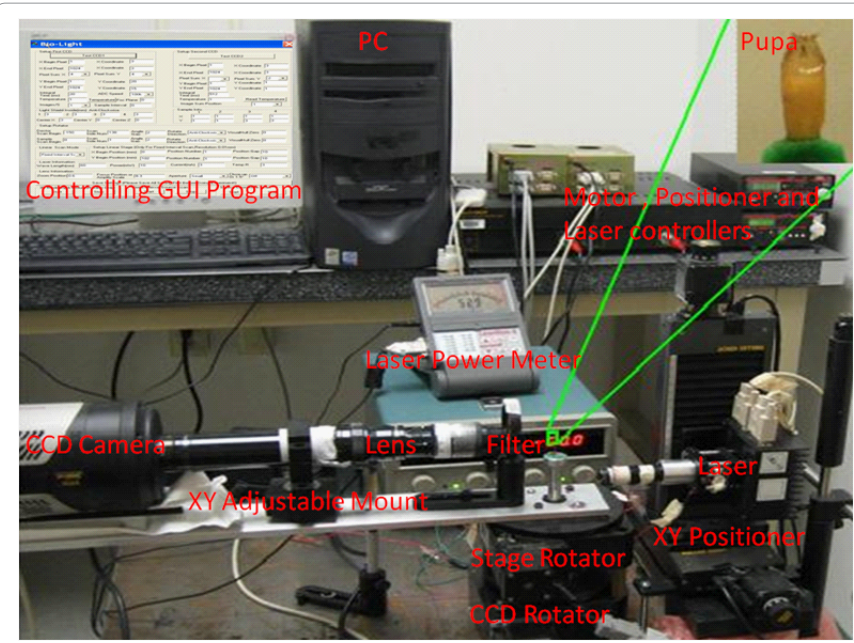

Figure 1: The 3D FMT in vivo imaging system. The insert is a close view of a meso-scale subject (Drosophila Pupa) in experiment.

*Corresponding authors: Yiyong Tan, J. Crayton Pruitt Family Department of Biomedical Engineering, University of Florida, Gainesville, FL 32611-6131, USA, Tel: 3522354188; E-mail: optictyy@ufl.edu

Huabei Jiang, J. Crayton Pruitt Family Department of Biomedical Engineering University of Florida, Gainesville, FL 32611-6131, USA, Tel: 352-2739336; E-mail hjiang@bme.ufl.edu

Received August 05, 2011; Accepted November 10, 2011; Published November 17, 2011

Citation: Tan Y, Jiang H (2011) A Fluorescence Molecular Tomography In-vivo Imaging System for Macro/Meso-Scale Subjects. J Biosens Bioelectron S4:001. doi: 10.4172/2155-6210.S4-001

Copyright: @ 2011 Tan Y, et al. This is an open-access article distributed under the terms of the Creative Commons Attribution License, which permits unrestricted use, distribution, and reproduction in any medium, provided the original author and source are credited. 
method, the CCD camera was modeled as a pinhole camera positioned in a coordinate system where its intrinsic and extrinsic parameters (i.e., space projective transformation matrices) were calibrated using the widely used chess board method, allowing the calculation of the extrinsic parameters for a new position while the intrinsic parameters stayed the same for all the positions [9]. A total of 72 projection images (one projection every $5^{\circ}$ by rotating $\mathrm{CCD} 360^{\circ}$ ) were used in our calculation giving a submillimeter resolution. The projection images were then converted into foreground/background binary silhouette images (the $2 \mathrm{D}$ projections of the $3 \mathrm{D}$ foreground subject). Each silhouette defined a back-projection of a cone that contained the geometrical information of the actual subject. Thus the overlapped volume of all 72 cones defined by silhouettes gave the 3D visual hull model of the arbitrarily shaped subject. A photoluminescent plate was used as the background screen which provided evenly distributed illumination and good contrast.

A 3D finite element mesh of the 3D model was then generated with the point cloud obtained by visual hull method: use point wrap method in Amira 3.1 to obtain the surface. Smooth and relaxation are applied to surface to improve mesh uniformity, and continuity which is the prerequisite of good $3 \mathrm{D}$ tetrahedron mesh generation to minimize mesh effect on final tomography reconstruction. Amira 3.1 (Visage Imaging Inc. San Diego, CA, USA) is used to generate 3D finite element mesh and optimize the mesh quality. The generated mesh is further optimized to decrease half band width (a pre-process to improve the numeric finite element computation memory efficiency and speed. The reconstruction computation results are same for with/without optimization of half band) for a fast memory efficiency reconstruction computation [10].

There is also a need to map the photon density read from the CCD camera (actually the virtual detector along the focal plane of the CCD camera) onto the arbitrarily shaped surface of the subject. We have adopted a method developed by Ntziachristos et al. that was able to realize the mapping accurately in their FMT studies $[11,12]$ In this method, a Lambert's cosine law and solid angle based light propagation model is established to correlate the photon density at the virtual detector and that at the animal surface. With this model, given the relative geometric relationship between the virtual detector and the subject surface, the numerical aperture of the virtual detectors and the visibility factor that can be experimentally calibrated, one can accurately convert the read out from the CCD into the photon density at the subject surface for tomographic reconstruction.

\section{Results}

\section{Macro-scale subject}

The recombinant amino terminal fragment (ATF) of uPA was produced from a bacterial expressing system using established protocol ATF peptides were then labeled with Cy5.5 dye and IR 800 dye which used to label breast cancer 4T1 cells [13]. After labeling, the intercellular dye and nonspecific binding are washed thoroughly. Cells were counted and specific numbers of cells $(200,000$ and 500,000) were injected into the haired Balb/C mouse for optical imaging, and the mouse was shaved before imaging. Experiments were conducted using a continuous wave $(\mathrm{CW})$ diode laser at $660 \mathrm{~nm}(25 \mathrm{~mW})$ as excitation and $710 \mathrm{~nm}$ band pass filter for Cy 5.5 emission light acquisition and $830 \mathrm{~nm}$ band pass filter for IR 800 emission light acquisition.

Since both dyes are labeled with recombinant amino terminal fragment (ATF) to improve stability and enable long time fluorescent monitoring, the stability difference between two dyes are not discernable. The variation in raw signals before and after 15 minute scan is within $5 \%$ and photo bleaching is not observed, therefore stability is very good to provide meaningful scan.

The injected tumor shape and volume may vary depending on the anatomic structure of the injection site and is roughly proportional to tumor cell number. Since the imaging agent are uniformly distributed
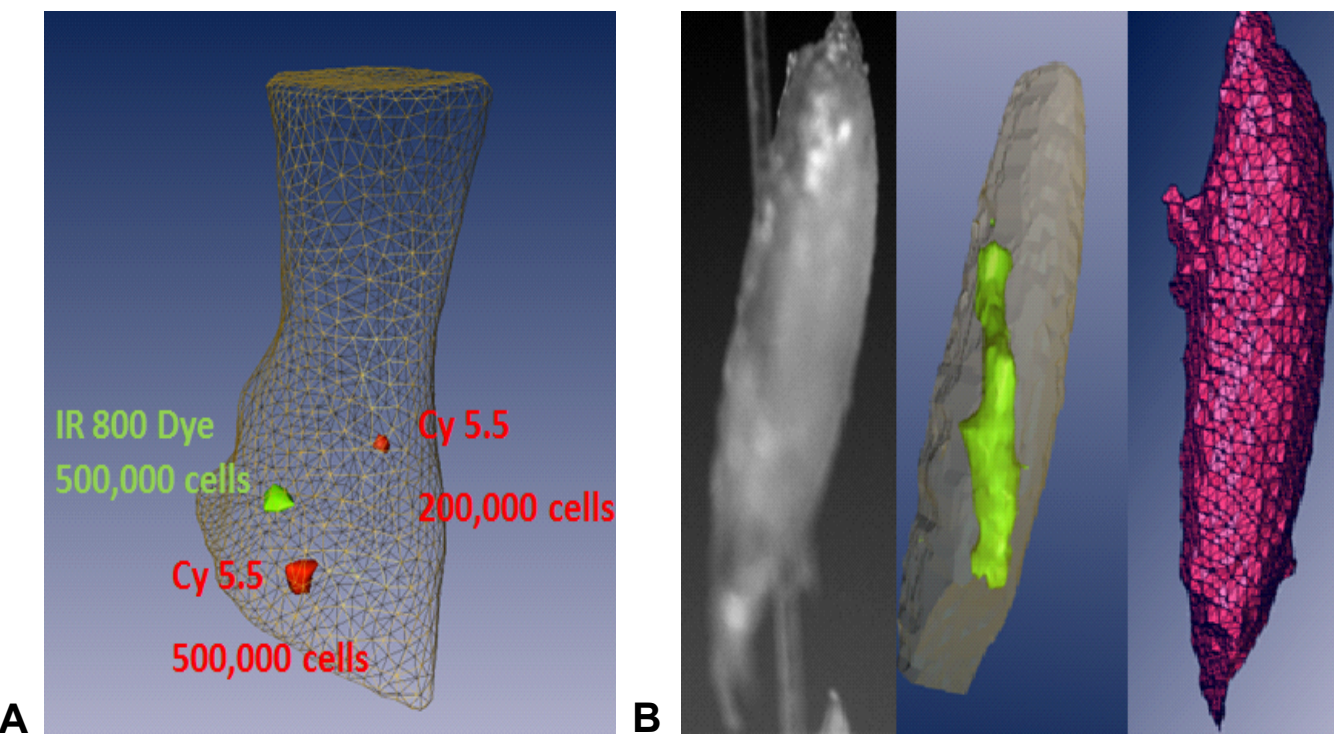

Figure 2: Reconstructed images of Marco-Scale and Meso-Scale subject:

A: Macro-Scale Subject Reconstructed image, using on diffusion model. The reconstructed images' isosurface is used to render 3D shell. 500,000 tumor cells imaged by IR 800 dye is reconstructed and rendered in green. 500,000 and 200,000 tumor cells imaged by Cy 5.5 are rendered in red.

B: Meso-Scale Subject Reconstructed Image. Left) Photograph of the pupa with microtube. Middle) reconstructed Cy5.5 dye-containing microtube. Right) 3D model and finite element mesh of pupa. 
in tumor cells, the reconstruction value is similar for different cell numbers, reconstructed target size is the better way to show different tumor cell numbers than absolute reconstructed value. Isosurface (70\% of maximum value) of the reconstructed fluorophore absorption coefficient is used to present the $3 \mathrm{D}$ reconstruction where the volume of the isosurface indicates the tumor cell number. The reconstructed tumor images is accurately reconstructed in terms of the volume (which is proportional to tumor cell number) and position (consistent with the exact tumor cell embedded positions and the localization error is within $3 \mathrm{~mm}$ ) as in Figure 2A shows.

\section{Meso-Scale Subject}

The experiments were conducted using a continuous wave (CW) $660 \mathrm{~nm}$ diode laser as excitation and $710 \mathrm{~nm}$ band pass filter for emission light acquisition. The 3D shape of the pupa was obtained with volume carving method based on silhouettes of 72 projections. Signals from a total of 484 sources at 4 CCD positions and 484 detector positions at the opposite side of the source location were collected. The $3 \mathrm{D}$ experimental data was mapped to 7 transverse slices and the $2 \mathrm{D}$ RTE based FMT algorithm was utilized for reconstruction.

The System is tested by meso scale pupa imaging experiment in other studies [14]. Briefly, a $3 \mathrm{~mm}$ long silica capillary microtube (outer diameter $=150 \mu \mathrm{m}$ and inner diameter $=100 \mu \mathrm{m}$ ) containing Cy 5.5 dye served as a target. The tube was inserted through the pupa as shown in Figure $2 \mathrm{~B}$. The Drosophila pupa was positioned vertically on a rotation stage. Laser beam was focused to source point at the pupa's shell with a plano-convex lens. The sample was rotated to 4 projection positions and transmitted light were captured with a $1024 \times 1024$ pixels CCD camera.

Since an early stage pupa is composed of mostly fat tissue like larva, the optical properties of the pupae can then be assumed homogenous. We also note that the images with the 2D RTE based FMT reconstruction algorithm give a target-localization error of up to $100 \mu \mathrm{m}[14,15]$. Figure $2 \mathrm{~B}$ shows the $3 \mathrm{D}$ rendering of the reconstructed images obtained from these 7 transverse slices.

\section{Discussion}

Compared to other imaging modalities like MRI, CT, PET etc, although quantitative accuracy and the resolution of Fluorescence molecular tomography (FMT) method need further improvement, FMT method is a more convenient and economic method while provides unique advantage for in vivo animal imaging with additional functional information for other methods such as high molecular specificity, nonionizing radiation, optical probe stability (no intensity decay over time like isotope) and the potential for simultaneous investigations of multiple targets using spectral probes without overlapped emission. In addition, FMT has high contrast, high specificity, and biggest reagent arsenal for almost every aspect of biology molecular events which has already been widely used for fundamental biology research and preclinical research.

In conclusion, a non contact full 360 degree FMT imaging scheme for macro-scale and meso/scale samples is investigated. The quantitative 3D non contact FMT system capable of revealing dye distribution for macro-scale and meso-scale animals in their natural states was built and

This article was originally published in a special issue, Biosensors: New Developments handled by Editor(s). Dr. Hahn Jong hoon, Pohang University of Science and Technology, South Korea; Dr. Yuehe Lin, Pacific Northwest National Laboratory, USA its performance is validated by experiments. While the results shown are encouraging, improvements are certainly needed especially with respect to the spatial resolution and data acquisition speed. To enable cell level event monitoring, the resolution needs to be improved from the current $100 \mu \mathrm{m}$ to $10 \mu \mathrm{m}$. In addition, high speed data acquisition will allow to realize real-time dynamic monitoring of biologic events. High speed sampling can be implemented with fast Galvo scanner for light delivery and EMCCD for data collection, making a frame rate of 100 possible.

\section{References}

1. Rao J, Dragulescu-Andrasi A, Yao H (2007) Fluorescence imaging in vivo: recent advances. Curr Opin Biotech 18: 17-25.

2. Leblond F, Davis SC, Valdés PA, Pogue BW (2010) Pre-clinical wholebody fluorescence imaging: Review of instruments, methods and applications. J Photoch Photobio B 98: 77-94.

3. Licha K, Olbrich C (2005) Optical imaging in drug discovery and diagnostic applications. Adv Drug Deliv Rev 57: 1087-1108.

4. Deliolanis NC, Dunham J, Wurdinger T, Figueiredo JL, Tannous BA, et al. (2009) In-vivo imaging of murine tumors using complete-angle projection fluorescence molecular tomography. J Biomed Opt 14: 030509

5. Deliolanis NC, Kasmieh R, Wurdinger T, Tannous BA, Shah K, et al. (2008) Performance of the red-shifted fluorescent proteins in deep-tissue molecular imaging applications. J Biomed Opt 13: 044008.

6. Tan Y, Jiang H (2008) Diffuse optical tomography guided quantitative fluorescence molecular tomography. Appl Opt 47: 2011-2016

7. Tan Y, Jiang H (2008) DOT guided fluorescence molecular tomography of arbitrarily shaped objects. Med Phys 35: 5703-5707.

8. Mulayim A, Yilmaz U, Atalay V (2003) Silhouette-based 3-D model reconstruction from multiple images. IEEE Trans Sys Man Cyber 33: 582-591.

9. Zhang Z (2000) A flexible new technique for camera calibration. IEEE T Pattern Anal 22: 1330-1334.

10. Press WA, Teukolsky SA, Vetterling WT, Flannery BP (2007) Numerical Recipes: The Art of Scientific Computing (3rd ed., Section 2.4) New York: Cambridge University Press, ISBN 978-0-521-88068-8.

11. Ntziachristos V, Graves E, Schultz R, Ripoll J (2004) Fluorescence molecular tomography: New detection schemes for acquiring high information content measurements. I S Biomed Imaging 2: 1475-1478.

12. Ripoll J, Schulz RB, Ntziachristos V (2003) Free-space propagation of diffuse light: Theory and experiments. Physical Review Letters 91:103901.

13. Yang L, Peng X, Wang Y, Wang X, Cao Z, et al. (2009) Receptortargeted nanoparticles for in vivo imaging of breast cancer. Clin Cancer Res 15 : $4722-4732$.

14. Tan $Y$, Novo $M$, Yao L, Zhou L, Jiang H (2011) In vivo monitoring of stem cells in Drosophila pupae using the radiative transfer equation-based fluorescence molecular tomography. Mol Imaging Biol 13: 868-873.

15. Joshi A, Rasmussen J, Sevick-Muraca E, Wareing T, McGhee J (2008) Radiative transport-based frequency-domain fluorescence tomography. Phys Med Biol 53: 2069-2088. 